\title{
GCU
}

Glasgow Caledonian

University

University for the Common Good

\section{Differentiating sitting and lying using a thigh-worn accelerometer}

Lyden, Kate; John, Dinesh; Dall, Philippa; Granat, Malcolm H.

Published in:

Medicine and Science in Sports and Exercise

DOI:

10.1249/MSS.0000000000000804

Publication date:

2016

Document Version

Author accepted manuscript

Link to publication in ResearchOnline

Citation for published version (Harvard):

Lyden, K, John, D, Dall, $\mathrm{P}$ \& Granat, MH 2016, 'Differentiating sitting and lying using a thigh-worn accelerometer', Medicine and Science in Sports and Exercise, vol. 48, no. 4, pp. 742-747.

https://doi.org/10.1249/MSS.0000000000000804

\section{General rights}

Copyright and moral rights for the publications made accessible in the public portal are retained by the authors and/or other copyright owners and it is a condition of accessing publications that users recognise and abide by the legal requirements associated with these rights.

Take down policy

If you believe that this document breaches copyright please view our takedown policy at https://edshare.gcu.ac.uk/id/eprint/5179 for details of how to contact us. 
Title: Differentiating Sitting and Lying Using a Thigh-Worn Accelerometer

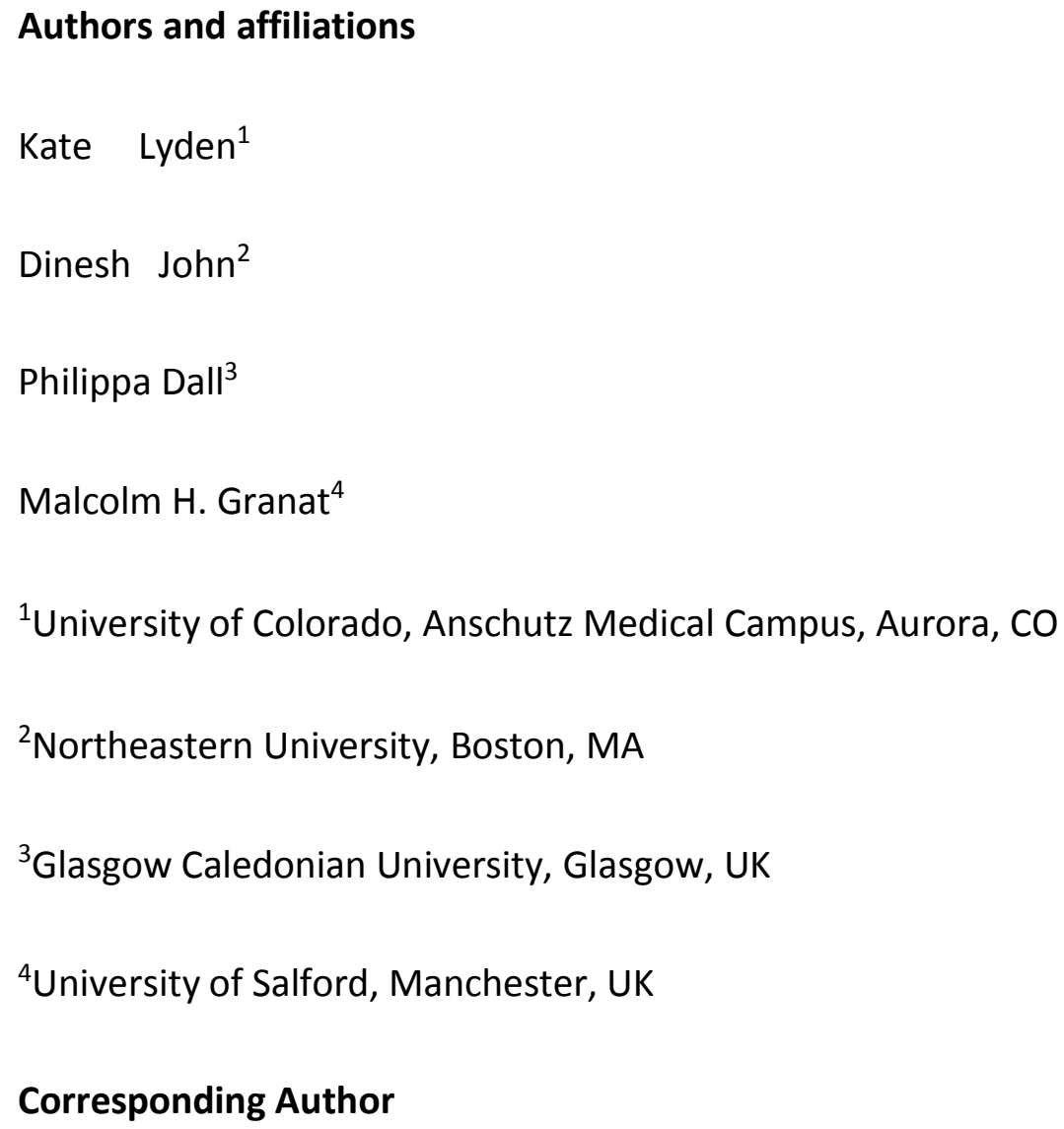




\section{Abstract:}

A tri-axial accelerometer worn on the thigh can provide information on the angle of rotation of the thigh. These data may be used to estimate periods of lying versus sitting. Purpose: To develop and test a classification algorithm to identify sedentary events as either lying or sitting events using a thigh-worn, tri-axial accelerometer. Methods: Seven day free-living activity from 14 sedentary workers was recorded using the activPAL3 ${ }^{\mathrm{TM}}$ monitor. Participants recorded when they went to bed and when they got up in a diary. All "in bed" sedentary events were assumed to be lying and all "not in bed" sedentary events as sitting. An algorithm computed the angle of rotation of the $y$-axis, which was used to detect orientation of the thigh. Crossing a rotational threshold in the transverse plane of $\pm 65^{\circ}$ was used to classify a sedentary event as lying. The classification accuracy of the algorithm was compared to self-reported classification from the diary. Results: The algorithm classified $96.7 \%$ of the sedentary time "in bed" (sensitivity) as lying and $92.9 \%$ of the time "not in bed" as not lying (specificity). Conclusion: Tri-axial accelerometer data recorded from a single site on the thigh can be used to classify sedentary events as sitting and lying. The automated method developed in this study will allow objective measurement of diurnal lying time and that while sleeping without relying on self-report. This will help advance the understanding of the impact of different types of sedentary behaviours on various health outcomes.

Keywords: activpal, sedentary, sitting, sleep, lying 


\section{Introduction}

Wearable sensors are used to collect continuous physiologic and/or kinematic information for extended periods of time (e.g. days to weeks) in community dwelling individuals. In physical activity and health research, these devices are typically used to estimate attributes of active behaviour (e.g. energy expenditure, time in moderate-to-vigorous physical activity) $(10,23)$. However, in light of emerging epidemiological, preclinical and clinical research, recent emphasis has been placed on developing devices and data processing methodologies that can also estimate attributes of sedentary behaviour (sitting and lying) $(2,10)$. A single device that can estimate various features of both active and sedentary behaviour in community dwelling individuals is necessary to understand how various components of habitual behaviour interact to affect health and disease etiology.

The activPAL3 ${ }^{\mathrm{TM}}$ (AP) (PAL technologies Ltd, Glasgow, UK) is a thigh-worn accelerometer-based activity monitor that detects static (gravity) and dynamic accelerations of the $x$-axis to detect sedentary (sitting and lying), standing and ambulatory events. Currently, the AP does not distinguish between sitting and lying events. However, because the AP is sensitive to acceleration from three orthogonal axes, it can also provide information about thigh rotation. During periods of lying, it is common for an individual to roll onto his/her side or stomach and such transitions in lying position alter the orientation of the sensor in the AP, which can be detected by changes in the acceleration signal from the $y$-axis The $y$-axis is in the same plane but perpendicular to the long axis of the femur (see Figure 1a). With these data, it may be possible to further differentiate sedentary behaviour as either sitting or lying. Therefore, the purpose of this study was to develop and test a classification algorithm to differentiate sedentary events as sitting and lying using acceleration data collected from a single AP activity monitor worn on the thigh. 


\section{Methods}

\section{Participants}

Data used to develop and validate the classification algorithm were obtained from a larger cross-sectional study, which measured seven days of objective physical activity and sedentary behaviour, and self-reported waking times in healthy working adults. Participants were community dwelling office employees from the Glasgow area of the UK aged 18-65 years. Fourteen participants were randomly selected for our study, which resulted in a total of 98 days and nights of data that were used to develop and validate the classification algorithm. The School of Health \& Life Sciences Ethics Committee, Glasgow Caledonian University, granted ethical approval for the study and written informed consent was obtained from participants.

\section{Measurement of physical behaviour}

Physical behaviour was measured using the AP worn on the midline of the thigh, midway between the hip and knee. The AP uses a digital tri-axial acceleration sensor (ADXL345, Analog Devices, Norwood, MA; $\pm 2 \mathrm{~g}$ ) to detect static and dynamic acceleration. The AP has excellent validity in differentiating among sitting/lying, standing and stepping $(13,15,19,21,25$, 26). Participants were given a waterproofed AP with instructions to wear it on their dominant thigh at all times for seven days (including overnight and during water-based activities) using a double-sided hypoallergenic pad. Each night, participants used a diary to record the time they went to bed at night and the time they got up in the morning. Total time "lying in bed" for each overnight period was computed as the difference between these two time points. If the monitor was removed from the thigh, participants recorded the times when the monitor was not worn. Non-wear data were not included in the analyses. 


\section{Algorithm development}

AP data were downloaded and post-processed using AP Professional Research Software v7.2.29 (PAL Technologies, Glasgow, Scotland). Both raw acceleration and event files were used in conjunction to develop the algorithm (14). The raw acceleration output file contains a digitized representation of the acceleration signal at an 8-bit resolution and a sampling rate of $20 \mathrm{~Hz}$. The event file contains classifications of 'sedentary,' 'standing' and 'stepping' events. Three participants were randomly selected from the sample of 14 to develop the algorithm. All participants in the development group had 7 days of continuous AP data ( 21 days), complete diaries, and did not report removing the monitor at any time during the recording period.

Raw acceleration files were post-processed using an algorithm developed in Matlab software (The MathWorks, Inc, Natick, MA). Briefly, each digitized sample of the raw acceleration signal from the AP was first converted to its equivalent g-force value (16) using ADXL345 specifications released publicly by the manufacturer (7), which translates to a linear scaling of the digital output within the $\pm 2 \mathrm{~g}$ dynamic range of the AP. The converted signal was low-pass filtered using a 20 -second moving average digital filter to smooth the signal by eliminating random noise that disrupt the static acceleration signal. An inverse sine function was used on the filtered output to compute tilt angle of the $y$-axis in radians, which was subsequently converted to degrees to yield an angle between $\pm 90^{\circ}$. Time-series graphical outputs of the rotational angles in each axis were visually compared to corresponding periods of "lying in-bed". Several arbitrarily selected threshold angles were tested to determine the one that returned the maximum classifications of lying events (event file) during the "lying in-bed" periods. A threshold of $65^{\circ}$ in the $y$-axis (where $0^{\circ},+90^{\circ}$ and $-90^{\circ}$ represented lying on one's back and on the right and left sides, respectively) yielded the highest classification accuracy in the development data. We did not use information from either the $\mathrm{x}$ or z-axes because it did not improve classification accuracy. 
angle equal to or between: $+65^{\circ}$ and $+90^{\circ}$ or $-65^{\circ}$ and $-90^{\circ}$ ), the algorithm recorded a crossing point value of '1.' When the signal subsequently fell below the threshold (i.e. an angle equal to or between: $+64^{\circ}$ and $-64^{\circ}$ ), a crossing point value of ' 0 ' was recorded. Time-stamped occurrences of threshold crossings were compared to corresponding time-stamped information from the event file. Any sedentary event with at least one crossing point of both 0 and 1 was classified as lying. The start and end times for lying in-bed were identified by the start and end times of the corresponding sedentary event (obtained from event file). Figure 1 (A) illustrates the directions of sensitivity of the tri-axial accelerometer within the AP when worn on the thigh in a sitting/supine position and (B) a cross-sectional depiction of the thigh indicating the $y$-axis's sensitivity to gravitational acceleration, the corresponding angles of rotation when lying supine and on either side, and the lying threshold angle of $65^{\circ}$.

\section{Algorithm validation}

The remaining 11 participants ( 77 days of AP data) were used as an independent validation sample. The algorithm was applied to continuous AP data for the total duration of measurement to determine the accuracy of the algorithm to detect lying in bed and its sensitivity and specificity during both lying in bed and "out of bed" periods. Participants did not self-report their behaviors (e.g. lying vs. non-lying) during out of bed periods, and thus all time out of bed was assumed to be non-lying.

Start and end times for self-reported lying in-bed were obtained from diaries and were visually compared to event files showing the time sequence of events over the course of each 24-hour period to determine if there were any substantial errors in self-report. Prior to validating the algorithm, we 'refined' the self-reported lying in-bed start and end times using the event file in conjunction with the self-report diaries. This was done to minimize errors 
attributable to self-report bias (e.g. rounding off: self-reporting 10:30 pm instead of 10:22pm). The following rules were used to refine the start times; 1 ) a duration of 15 minutes was considered as an acceptable error for self-reported lying in-bed start and end times, 2) if the selfreported lying in-bed start time was within an upright event, the start time of the next sedentary event was considered to be the refined lying in-bed start time, 3 ) if the self-reported lying in-bed start time was within a sedentary event but was more than 15 minutes before the next upright event then the start-time of the sedentary event was used as the refined lying in-bed start time, and 4) if the self-reported lying in-bed start time was within a sedentary event but within 15 minutes of the next upright event, then the start of the subsequent sedentary event was considered as the refined lying in-bed start time. The following rules were used to refine the end times; 1) If the self-reported lying in-bed end time was within a sedentary event then the end of that sedentary event was used as the lying in-bed end time, and 2) if the self-reported lying in-bed end time was within an upright event then the end of the previous sedentary event was considered as the refined lying in-bed end time.

Refined self-reported times were determined for each of the 77 days of data in the validation group and were compared to estimates obtained from our algorithm using prediction bias and precision $(95 \% \mathrm{Cl})$. We also computed the sensitivity and specificity of the algorithm in correctly identifying time lying in bed. Sensitivity of the algorithm was defined as the ratio of the total time classified as lying in-bed by our algorithm to the total self-reported lying in bedtime. Specificity of the algorithm was defined as the ratio of the total time sedentary when not lying inbed that was not classified as lying by the algorithm to the total time sedentary when not lying inbed.

\section{Results}


All data are presented as mean \pm SD unless otherwise noted. Our sample consisted of 3 males and 11 females (age $=48 \pm 9$ years; $\mathrm{BMI}=26.6 \pm 2.7 \mathrm{~kg} / \mathrm{m}^{2}$ ) who continuously wore the AP for an average of $120.8 \pm 25.9$ hours. The mean time self-reported as lying and not lying was 53.6 \pm 14.5 and $67.1 \pm 11.4$ hours per recording period, respectively.

The duration of time in bed detected by our algorithm was similar to that reported previously using a combination of self-report and accelerometry (20). Figure $\mathbf{2}$ shows the total hours per recording period estimated as lying in comparison to self-reported lying for the development (nos.1-3) and validation (nos. 4-14) participants. For the validation group, mean time estimated as lying by the algorithm was $50.9 \pm 8.9$ hours and mean self-reported lying time was $54.8 \pm 10.4$ hours. This resulted in a small bias $(95 \% \mathrm{Cl})$ of $-3.9(-0.63,8.4)$ hours, or $36.2 \mathrm{~min}$ per night. Additionally, when applied to 24 hours of continuous data, the algorithm demonstrated high ( $\geq 90 \%$ ) sensitivity (range: 76.4 to $99.2 \%$ ) and specificity (range: 87.6 to $99.9 \%$ ) in correctly classifying a sedentary event as lying (figure 3).

During self-reported lying time, the average length of sedentary events classified by the algorithm as lying was considerably longer $(5.4 \pm 2.1$ hours) than those classified as not lying $(0.3 \pm$ 0.3 hours). However, during self-reported not lying time, the mean duration of sedentary events classified as lying and not lying were relatively short ( $0.9 \pm 0.7$ and $0.2 \pm 0.1$ hours, respectively). During self-reported lying time, the lying threshold $\left( \pm 65^{\circ}\right)$ was crossed approximately 5 to 17 times during sedentary events classified by the algorithm as lying. During self-reported not lying time, the lying threshold $\left( \pm 65^{\circ}\right)$ was crossed approximately 1 to 6 times during sedentary events classified by the algorithm as lying.

\section{Discussion}

Main Findings 
Detecting lying in bed: This is the first study to demonstrate that acceleration data from a single sensor on the thigh can be used to accurately distinguish sedentary events as sitting or lying. The algorithm developed and validated in this study used the acceleration signal from the $y$-axis (medial-lateral plane) of a thigh-worn AP to determine rotation of the thigh. Using a threshold of $\pm 65^{\circ}$, the algorithm correctly identified self-reported lying for $96.7 \pm 2.8 \%$ of the time.

In general, we observed similar patterns across participants during the lying in bed periods. Lying in bed periods often comprised of few but long sedentary events, which resulted from the occurrence of short upright events in between. Occasionally the short upright events were also separated by a short sedentary event. These patterns are consistent with what we would expect if an individual were to briefly wake up from sleep to get a drink of water or to use the bathroom. Thus, instances when the algorithm falsely classifies lying in bed as sitting could actually be brief sitting events that were not captured by the self-report diaries. Conversely, if an individual is in the lying posture, but does not rotate their thigh beyond the $65^{\circ}$ threshold (i.e. flat on their back) at any point during the sedentary event, the current algorithm will misclassify the event as sitting. However, most individuals typically change positions (back, side, or stomach) up to 45 times/night over an 8-hour sleep period (17). More than $90 \%$ of these transitions occur with 10 to 15 minutes of each other and it is very rare for a position to be held for more than one hour (17). These lying patterns typically tend to be consistently repeated over several nights (17). Additionally, more sophisticated statistical modeling using features from the 3-axes acceleration signal in combination with our lying threshold may reduce estimation errors.

Detecting lying out of bed. The algorithm developed in this study can also be used to detect lying during waking periods. Most studies currently use self-report to determine the duration of lying at night $(18,25)$ and do not provide information on daytime lying/sleep, which may be considerably shorter than that at night and more interspersed during the day. Due to the inability 
of the current AP software to distinguish between sitting and lying, studies using the AP to objectively measure sedentary behaviour mostly assume that sedentary behaviour recorded during the day represents only sitting. This assumption may have additional implications on associations between sedentary behaviour and health. The methodology developed in our study can be used to distinguish between diurnal sitting and lying behaviour.

We did not provide specific instructions to record lying time during the day. Thus, we calculated wake time as the period that was not reported as lying in-bed and assumed that waking periods did not consist of any lying bouts. Therefore, a portion of the sedentary events registered by the AP as sitting may actually be lying. Additionally, most of the out of bed sedentary events classified as lying occurred in the evening, close to when the individual reported having gone to bed. This suggests that the individual may either have inaccurately reported the start of the in bed period or may have been lying on a couch or in bed prior to lying in bed to sleep.

Lying events (both in and out of bed) where the threshold is not breached at least once will be misclassified as sitting. Such instances are more likely to occur during short lying events compared to longer events. The longer an individual lies, the more opportunity there is for them to "adjust" their lying position and breach the threshold at least once during that event. While lying out of bed events are likely to be shorter than lying in bed, we do not anticipate that these events will be predominantly characterised as lying without position adjustment. Potential factors that contribute to minimizing such instances are the physiological responses to tissue compression and a greater sensitivity to these physiological responses due to wakefulness (or likelihood of less intense sleep). It is likely that an individual will respond much earlier to the onset of musculoskeletal discomfort when lying and awake as compared to when in deep sleep. Nonetheless, measurement error will occur occasionally, however it is likely be marginal in the context of estimating lying time over a period of 24-hours. 


\section{Practical Implications}

Improving human behaviour assessment: Research participants are often asked to wear activity monitors for 24 hours per day, over multiple days in order to capture detailed estimates of habitual activity and sedentary behaviour. Sedentary behaviour is defined as sitting or lying whilst awake (27), and differentiating between waking and sleeping during 24 hour monitoring is currently a methodological challenge in the assessment of sedentary behaviour (10). Our method may allow for a clearer distinction between physical activity, sedentary behavior, and lying while asleep during such investigations and ultimately a better understanding of the dose-response relationship between these behaviors and health. Although lying is not synonymous with sleep, the algorithm developed in this study provides valuable information that may prove useful to future methods aimed at estimating sleep.

Duration of sleep is suggested to be associated with chronic conditions including type II diabetes, respiratory disorders, cardiovascular disease, and obesity and morbidity and mortality $(3,6,9,11,12)$. For example, chronic sleep deprivation may increase the risk for obesity by increasing fatigue leading to decreased physical activity and increased sedentary behavior and by activating neuro-hormonal pathways that increase appetite (e.g. leptin, ghrelin) and caloric intake $(8,24,28)$. Thus, objectively measuring total lying time while asleep during surveillance and intervention research is essential to determine the relationship between daily habitual behavior and health. Accurate estimates of physical activity, sedentary behavior and sleep durations will not only allow the quantification of the independent and synergistic effect of these behaviors on health, but may also provide valuable insight on tailoring interventions to target and modify these individual behaviors to optimize health outcomes.

Improving energy expenditure estimation: Accurate estimates of energy expenditure may 
be necessary to establish causal relationships between physical behaviors and health outcomes. The Compendium of Physical Activities reports the energy requirements of sleeping, lying down and sitting to be approximately $0.90,1.0$ and $1.30 \mathrm{METs}$, respectively (1). Given that an individual engages in lying and sitting for a major portion of the 24-hour day (22), distinguishing between lying, sitting and sleeping will help to further refine the estimation of daily energy expenditure. Increasing feasibility of long-term monitoring: The accuracy of existing methods to distinguish lying from sitting increases with the number of sensors worn at multiple locations on the body. The IDEEA monitor, which uses five acceleration sensors placed on different parts of the body can correctly classify lying with almost $100 \%$ accuracy in a laboratory setting (29) and similar findings have been reported with other multi-sensing devices (4). A recent study used 2 timesynchronized AP monitors, one on the thigh and the other on the torso, to detect the precise times when a person transitions from an upright or sitting position to the lying position (5). Drawbacks to the abovementioned multi-sensor methodologies are associated with the feasibility of long-term behavior monitoring in the free-living environment. Form-factor and technical limitations including connecting cables, short battery life (3-15 hours) to sustain wireless transmission, need for advanced computational resources, and physical discomfort increases both participant and researcher burden and thereby compromises wear-time and data quality/loss. The high accuracy of our methodology in community dwelling adults over a relatively long period of time using a single thigh-worn AP is a potential improvement over existing methods to distinguish among physical activity, lying, and sitting in the free-living environment.

Limitations: The main limitation of this study is our use of self-report diaries to identify "lying in bed" periods and our assumptions that all sedentary events during "lying in bed" time corresponds to lying and all sedentary events during "out of bed" time corresponds to sitting. This may result in misclassification of some of the algorithm estimated lying time, which may have compromised the sensitivity of our algorithm (e.g. subjects 4 and 10 in Fig3). We anticipate that 
validating the algorithm using a more robust criterion (e.g. direct observation) may provide a true representation of the accuracy of the algorithm to distinguish between sitting and lying for a 24hour duration. A second limitation is our small sample. However, despite the small training $(\mathrm{N}=$ 3) and validation ( $N=11$ ) groups, each participant was studied for seven continuous days, resulting in 2,352 hours of accelerometer data sampled at $20 \mathrm{~Hz}$. Although our sample was predominantly female, we do not believe this limits the generalizability of the results. In general, men have different body shapes than women, however, we don't expect the method to be impacted significantly by gender so long as placement of the AP is standardized to the anterior portion of the thigh. The lying threshold is conservative and will account for minor discrepancies attributable to anthropometrics. Similarly, we do not believe the algorithm threshold is biased by behavioural characteristics unique to our sample, however validation in a more diverse sample would be beneficial.

\section{Conclusion}

This proof of concept study demonstrates that accelerometer data from a commonly used activity monitor can be used to accurately distinguish between sitting and lying postures more than $96 \%$ of the time. Future work will use a more robust criterion, and sophisticated modeling techniques to improve the current algorithm in a larger simple with varying anthropometric and demographic characteristics. While we examined the use of dual and triaxial signals in detecting lying in our sample and found no improvement in classification accuracy, future work could explore this further in a larger and diverse sample. While sensor response characteristics may yield subtle yet inconsequential differences between the lying thresholds when using dual or triaxial acceleration, there will be a greater demand for computational resources to process multiaxial signals that may impact analyses efficiency. We anticipate that the concepts developed and tested in the current study will inform the development of new methods to estimate detailed 
features of sleep from wearable accelerometers.

\section{Acknowledgements}

Paragraph 24 The authors would like to thank all participants who volunteered to participate in this study. Current addresses and affiliations: Kate Lyden is now a Biomedical Research Associate at Misfit, Inc (Burlingame, CA).

Conflict of Interest

Paragraph 25 The results of the present study do not constitute endorsement by ACSM. Malcolm Granat is a co-inventor of the activPAL and director of PAL Technologies Ltd that manufactures the activPAL devices used in this study. No funding was provided to this study by PAL Technologies Ltd. The remaining authors declare no competing interests.

\section{References}

1. Ainsworth BE, Haskell WL, Herrmann SD et al. 2011 Compendium of Physical Activities: a second update of codes and MET values. Medicine and science in sports and exercise. 2011;43(8):1575-81.

2. Atkin AJ, Gorely T, Clemes SA et al. Methods of Measurement in epidemiology: Sedentary Behaviour. International journal of epidemiology. 2012;41(5):1460-71.

3. Ayas NT, White DP, Al-Delaimy WK et al. A prospective study of self-reported sleep duration and incident diabetes in women. Diabetes care. 2003;26(2):380-4.

4. Bao L, Intille SS. Activity recognition from user-annotated acceleration data. In. Pervasive Computing: Springer; 2004, pp. 1-17. 
5. Bassett DR, Jr., John D, Conger SA, Rider BC, Passmore RM, Clark JM. Detection of lying down, sitting, standing, and stepping using two activPAL monitors. Medicine and science in sports and exercise. 2014;46(10):2025-9.

6. Cizza G, Skarulis M, Mignot E. A link between short sleep and obesity: building the evidence for causation. Sleep. 2005;28(10):1217-20. Analog Devices Web site [Internet]: Analog Devices; (cited 2015 Oct 17) Available from:

http://www.analog.com/en/products/mems/mems-accelerometers/adxl345.htmlproduct-overview.

7. Dinges DF, Pack F, Williams $\mathrm{K}$ et al. Cumulative sleepiness, mood disturbance, and psychomotor vigilance performance decrements during a week of sleep restricted to 4-5 hours per night. Sleep. 1997;20(4):267-77.

8. Ferrie JE, Shipley MJ, Cappuccio FP et al. A prospective study of change in sleep duration: associations with mortality in the Whitehall II cohort. Sleep. 2007;30(12):1659-66.

9. Freedson P, Bowles HR, Troiano R, Haskell W. Assessment of physical activity using wearable monitors: recommendations for monitor calibration and use in the field. Medicine and science in sports and exercise. 2012;44(1 Suppl 1):S1-4.

10. Gangwisch JE, Heymsfield SB, Boden-Albala B et al. Short sleep duration as a risk factor for hypertension: analyses of the first National Health and Nutrition Examination Survey. Hypertension. 2006;47(5):833-9.

11. Gangwisch JE, Malaspina D, Babiss LA et al. Short sleep duration as a risk factor for hypercholesterolemia: analyses of the National Longitudinal Study of Adolescent Health. Sleep. 2010;33(7):956-61.

12. Godfrey A, Culhane KM, Lyons GM. Comparison of the performance of the activPAL Professional physical activity logger to a discrete accelerometer-based activity monitor. 
Medical engineering \& physics. 2007;29(8):930-4.

13. Granat MH. Event-based analysis of free-living behaviour. Physiological Measurement. 2012;33(11):1785.

14. Grant PM, Ryan CG, Tigbe WW, Granat MH. The validation of a novel activity monitor in the measurement of posture and motion during everyday activities. British Journal of sports medicine. 2006;40(12):992-7.

15. John D, Freedson P. ActiGraph and Actical physical activity monitors: a peek under the hood. Medicine and science in sports and exercise. 2012;44(1 Suppl 1):S86-9.

16. Johnson $\mathrm{H}$, Swan T, Weigand $\mathrm{G}$. In what positions do healthy people sleep? Journal of the American Medical Association. 1930;94(26):2058-62.

17. Knutson KL, Lauderdale DS. Sociodemographic and behavioral predictors of bed time and wake time among US adolescents aged 15 to 17 years. The Journal of Pediatrics. 2009;154(3):426-30, 30 e1.

18. Kozey-Keadle S, Libertine A, Lyden K, Staudenmayer J, Freedson PS. Validation of wearable monitors for assessing sedentary behavior. Medicine and science in sports and exercise. 2011;43(8):1561-7.

19. Lauderdale DS, Knutson KL, Yan LL et al. Objectively measured sleep characteristics among early-middle-aged adults: the CARDIA study. American journal of epidemiology. 2006;164(1):5-16.

20. Lyden K, Kozey-Keadle SL, Staudenmayer JW, Freedson PS. Validity of two wearable monitors to estimate breaks from sedentary time. Medicine and science in sports and exercise. 2012;44(11):2243-52.

21. Matthews CE, Chen KY, Freedson PS et al. Amount of time spent in sedentary behaviors in the United States, 2003-2004. American journal of epidemiology. 2008;167(7):875-81. 
22. Matthews CE, Hagstromer M, Pober DM, Bowles HR. Best practices for using physical activity monitors in population-based research. Medicine and science in sports and exercise. 2012;44(1 Suppl 1):S68-76.

23. Patel SR, Malhotra A, White DP, Gottlieb DJ, Hu FB. Association between reduced sleep and weight gain in women. American journal of epidemiology. 2006;164(10):947-54.

24. Reynolds CF, 3rd, Serody L, Okun ML et al. Protecting sleep, promoting health in later life: a randomized clinical trial. Psychosomatic medicine. 2010;72(2):178-86.

25. Ryan CG, Grant PM, Tigbe WW, Granat MH. The validity and reliability of a novel activity monitor as a measure of walking. British journal of sports medicine. 2006;40(9):779-84.

26. Sedentary Behavior Research Network. Letter to the editor: standardized use of the terms" sedentary" and" sedentary behaviours". Applied physiology, nutrition, and metabolism. 2012;37(3):540.

27. Spiegel K, Tasali E, Penev P, Van Cauter E. Brief communication: Sleep curtailment in healthy young men is associated with decreased leptin levels, elevated ghrelin levels, and increased hunger and appetite. Annals of internal medicine. 2004;141(11):846-50.

28. Zhang K, Werner P, Sun M, Pi-Sunyer FX, Boozer CN. Measurement of human daily physical activity. Obesity research. 2003;11(1):33-40.

\section{Figure Captions}

Figure $1(A)$ : The direction of sensitivity of the $x, y$ and $z$-axes of the accelerometer in the AP when the device is worn on the thigh and when the person is sitting or supine Figure $1(B)$ : Cross-section of the thigh depicting the thresholds of the rotational angle $\left( \pm 65^{\circ}\right)$ of the $y$-axis that classify sedentary events as lying. Rotational angle of $0^{\circ}$ indicates sitting/lying on the back when the $y$-axis is oriented in the plane of the horizon (i.e. acceleration $=0 \mathrm{~g}$ ). Rotational 
angles of $\pm 90^{\circ}$ indicates lying on the left or right sides when the $y$-axis is orthogonal to horizon (i.e. acceleration $=1 \mathrm{~g}) . \mathrm{a}=$ acceleration.

Figure 2: Total hours per recording period estimated as lying in comparison to self-reported lying for the development (participants 1-3) and validation (participants 4-14) groups.

Figure 3: Sensitivity and specificity of the algorithm to distinguish sedentary events as sitting or lying when applied to continuous 24-hour data. 
Figure 1

A)

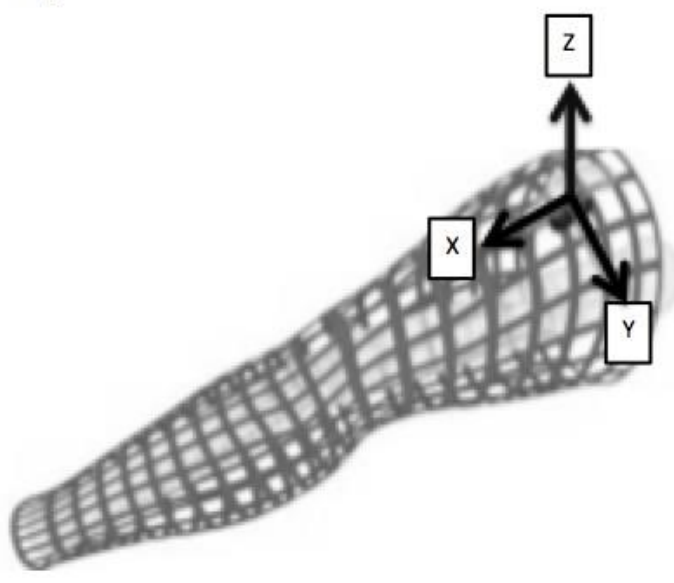

B) Lying on right side $+90^{\circ}(\mathrm{a}=1 \mathrm{~g})$
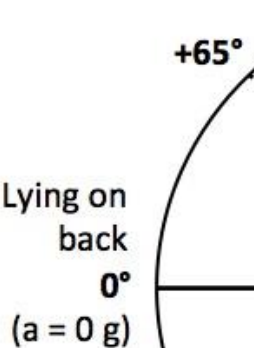
)

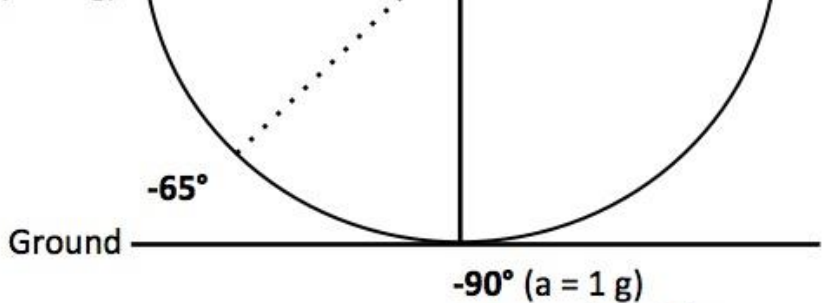

Lying on left side 
Figure 2

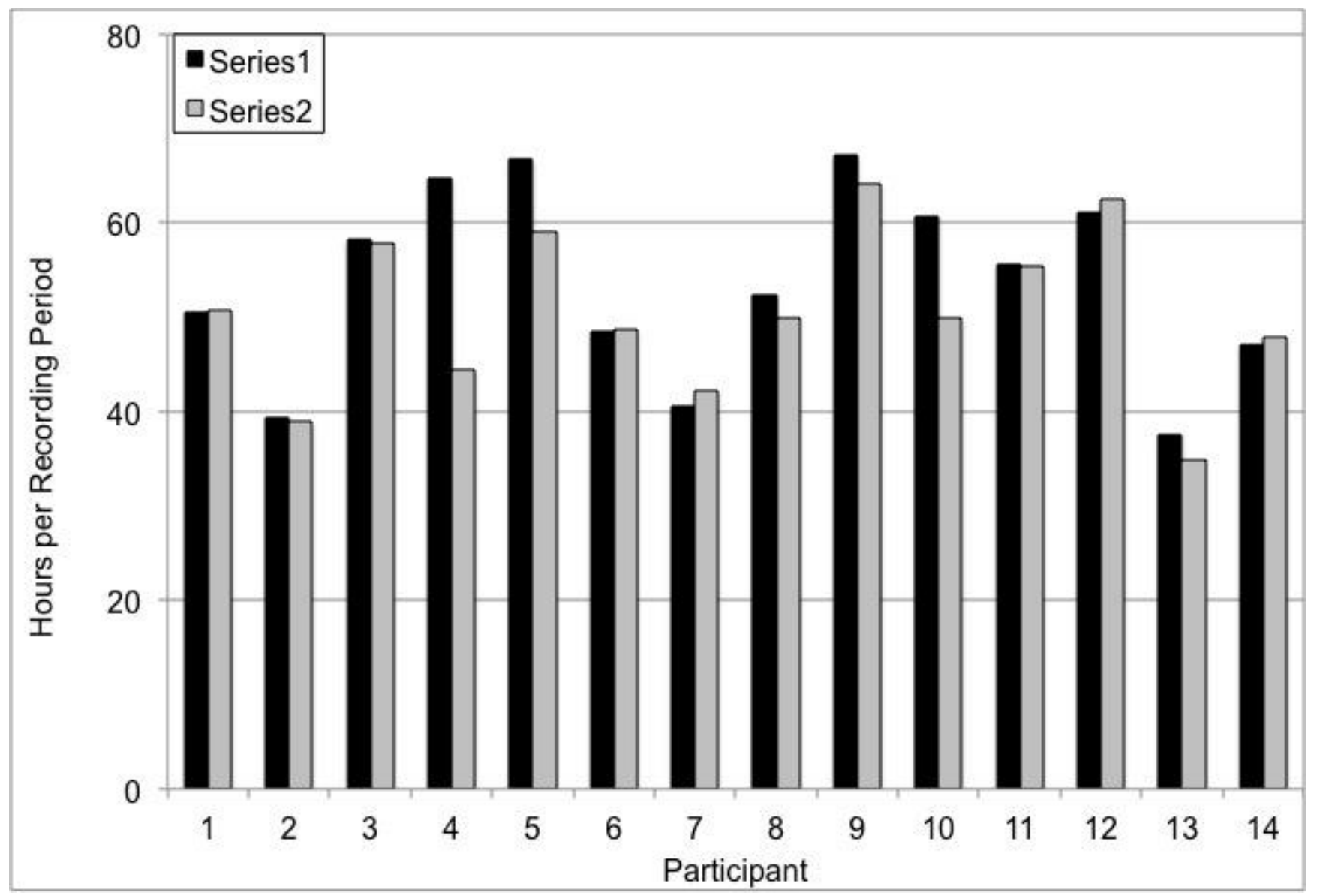


Figure 3

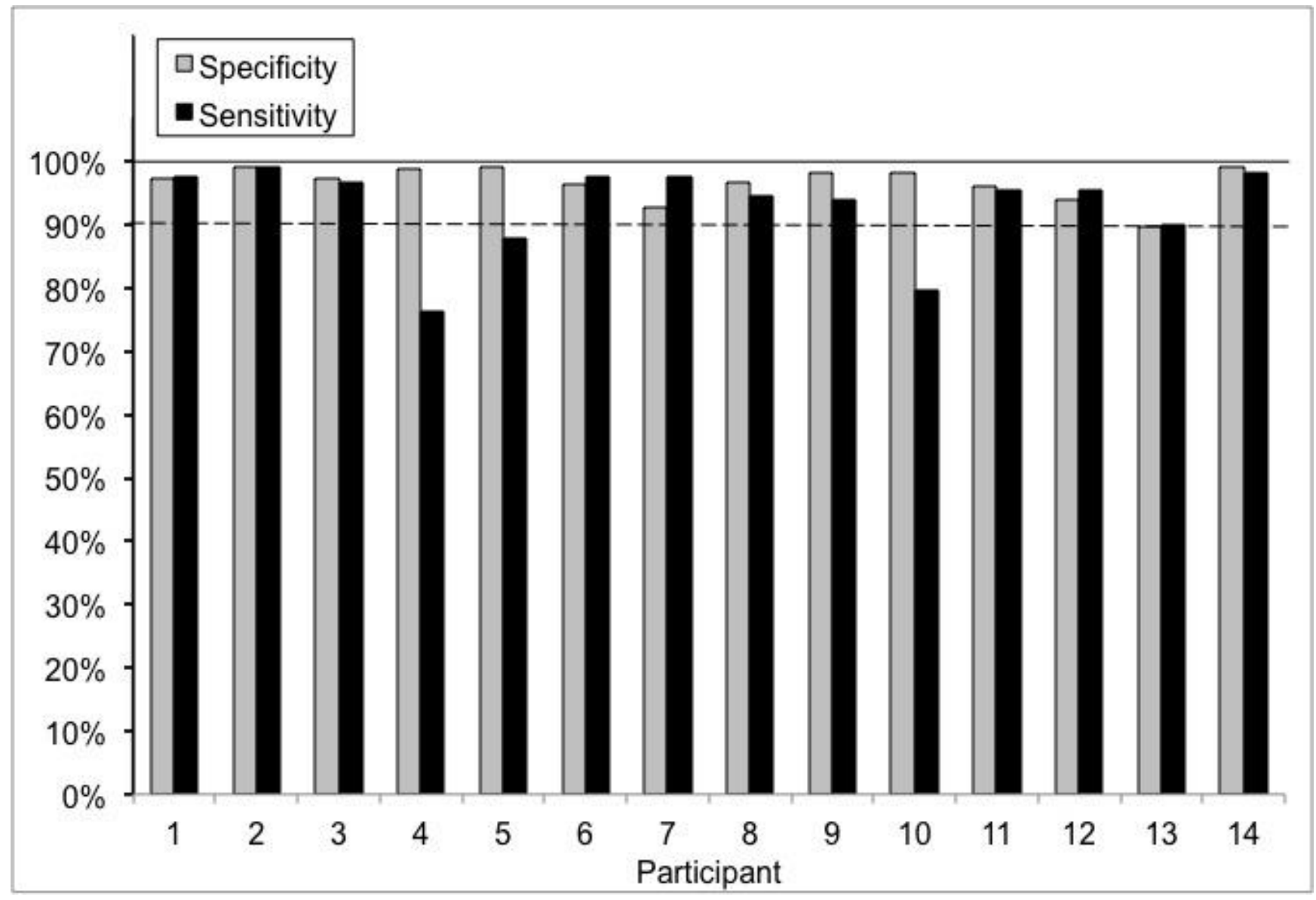

